\title{
New theories expand cognition to fungi
}

Consciousness is an elusive concept. Professor Nicholas Money of Miami University in Oxford, Ohio has argued for a new philosophy of cellular consciousness and suggests that fungi have minds. When we explore the sensitivity and recognise their ability to interpret and respond to their environment, it seems logical to extend the definition of consciousness to species without nervous systems. Fung show remarkable abilities in their responses to external stirili. Recent research on the reshaping how we think about microorganisms and ourselves.

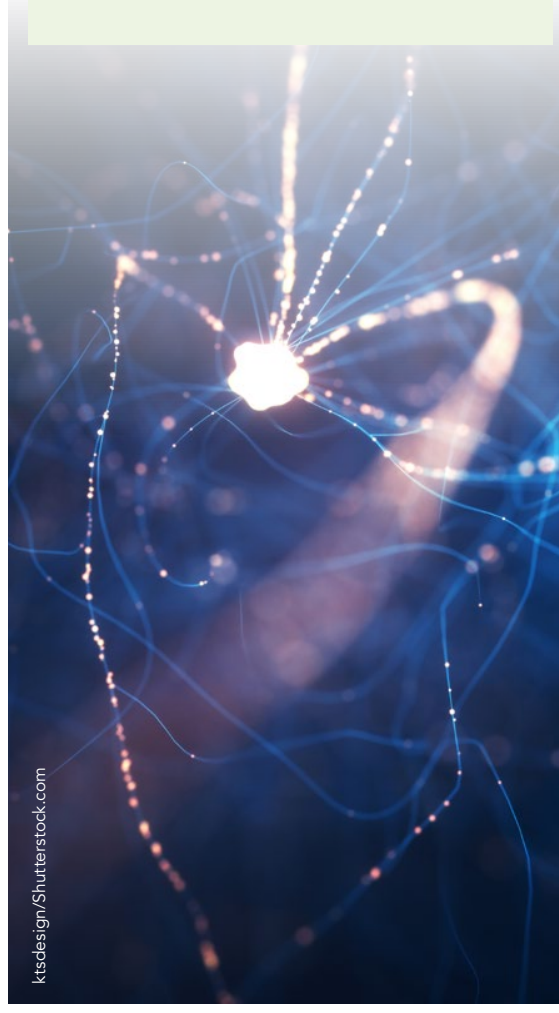

wareness is a complicated thing and consciousness is more that the brain is an organ for thousands of years and may have had a rudimenta idea of its function. The ancients would have observed changes in behaviour following head injuries, or perhaps they for instantly slaying their enemies. In our time, the way that the brain controls our actions and personalities remain matters of intense debate. Although the study of behavioural science has been extended to species without complex brains, most scientists believe that som form of nervous system is essential for an organism to possess consciousnes It was only in the last century that a ew investigators began to re-evaluate their definitions of intelligence and consciousness and examine whether brainless organisms could be sidered conscious.

It is difficult to define consciousness in away that is fair to every type of and qualifications which make up our understanding of consciousness, we must look beyond our human-centred worldview. For example, if we assume mind can only exist in a creature capable of movement, plants and fungi could. never be conscious. Likewise, if we believe that consciousness must entail networks of cells which form patterns within a more complex body, this will exclude single-celled organisms.

It was these unconventional interpretations which sparked the first inquiry into non-human consciousness. In the early 1900 s, scientists began to think of consciousness at the cellular level. This led to a model of consciousness which were broadly grounded in the biology of the cell. Initial research into amoeba behaviour, for example, demonstrated how single-celled organisms could react to stimuli, escape predation, and move toward food. They even expressed forms of cooperative behaviour. In one changes in the hunting patterns of amoebas as they pursued their single celled prey-When surrounded by abundant food, the amoeba was less active, but when the same food was scattered more sparsely, the amoeba sensed the need to change its behaviour and became more active in its search.

These experiments helped shape the concept of minimal selthood, a pattern of conscious behaviour that does not require the organism to possess a brain or neural tissue. Instead, minimal selfhood is based on three intrinsically reflective activities: self-maintenance, self-reproduction, and self-containment. An organism is said directe ens min to mantain its procan reproduce treff, and is defined by a physical boundary.

\section{THE FUNGAL BRAIN}

Professor Money is interested in the philosophy of consciousness when applied to fungal behaviour. Like the amoebas, fungal hyphae (the microscopic threads which make up the body of a fungus) react to their environment, pursue food, and recognise a threat. Money recognised that they embody the concept of minimal selfhood without employing any nervous system, because their filamentous hyphae and their colonies or mycelia operate as it they possess a very colmple form of intelligence. The fungal colony, in other words, can be seen as
an organism with a primitive mind. This

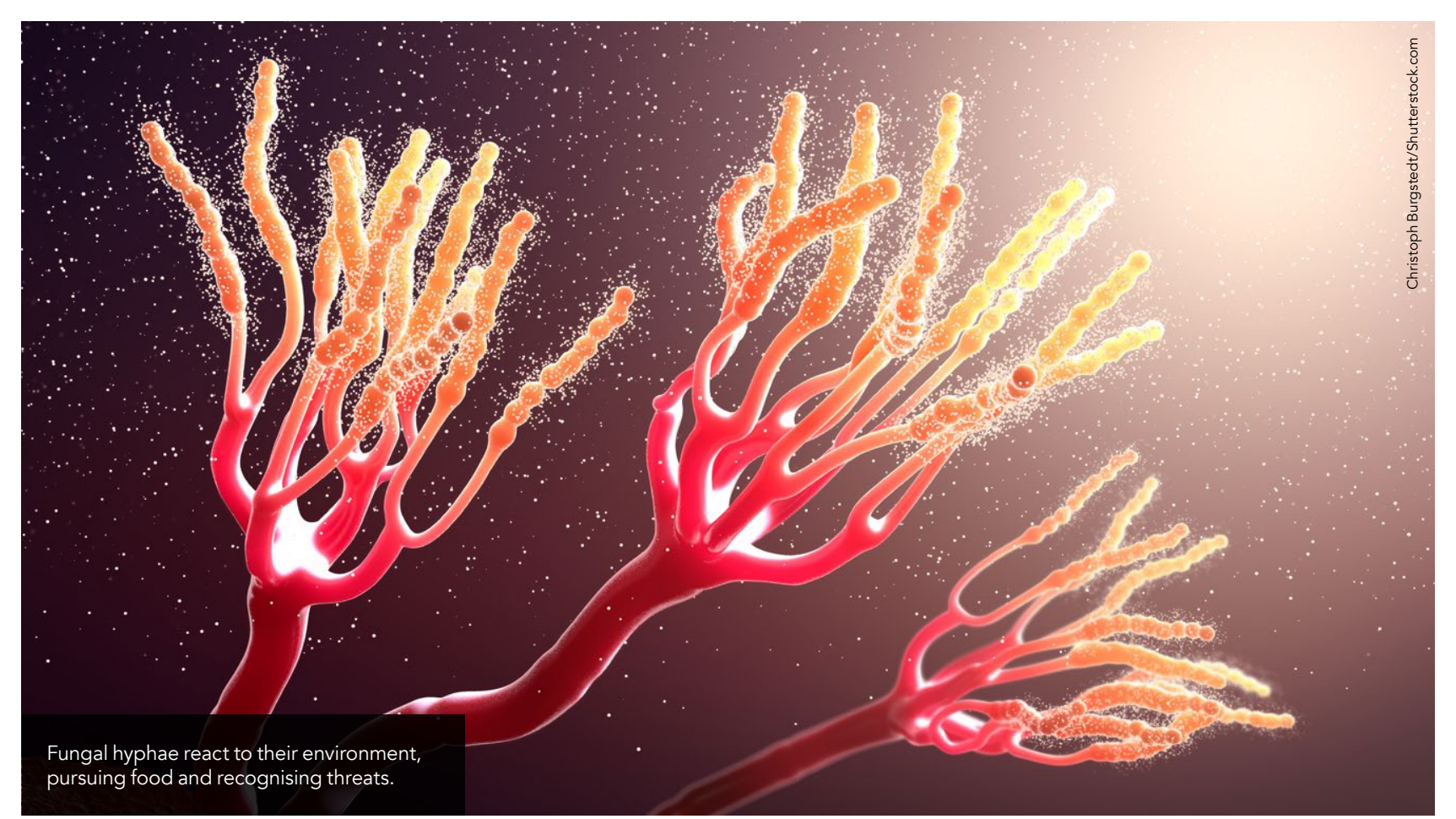

functions at the cellular level when the Fungi deploy these little spheres to $\begin{array}{lll}\text { threads elongate at their tips in response } & \text { repair and patch injuries similar to the } \\ \text { to different stimuli. Each of these threads, } & \text { way blood clots in animals. The hyphae the unique growth patterns of } \\ \text { fungi, which differ from species to species. }\end{array}$ which is adapted for the invasion and are also sensitive to microscopic ridges, digestion of

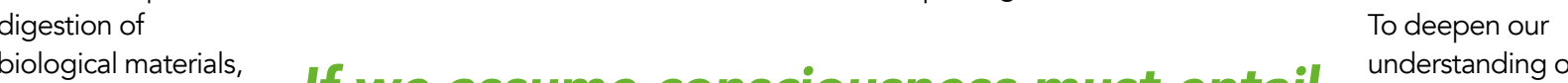
produces thousands If we assume consciousness must entail understanding of of internal vesicles networks of cells which form patterns need to look at (fluid-filled spheres mycelia in their natura enclosed by a within a more complex body, this will habitats, where the $\begin{array}{lll}\begin{array}{l}\text { membrane) every } \\ \text { minute. The }\end{array} & \text { exclude single-celled organisms. } & \text { fungal "brain" has the } \\ \text { opportunity to respond } \\ \text { to the richness of the }\end{array}$ vesicles travel to which helps some pathogenic fungi the surface of the filaments, delivering which helps some pathogenic fungi
the materials for elongation. The rapid detect the openings on the surface of motion and complex organisation of and consciousness of the fungus. to the richness of the

The vesicle mobilisation mechanism can the leaves of their host plants. Responses forest floor rather than the featureless expands, it detects the physical structum ofits surroundings and responds to the avalability of food and the presence of are punctured or sliced in the laboratory.

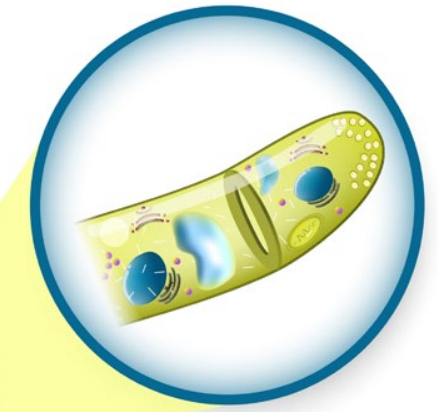
plants and other organisms. The overall pattern of branching is determined by the genetic code of the fungus, but the exact positions of each branch are dictated by the microscopic character of the environment. For this reason, the precise shape of each fungal colony is never

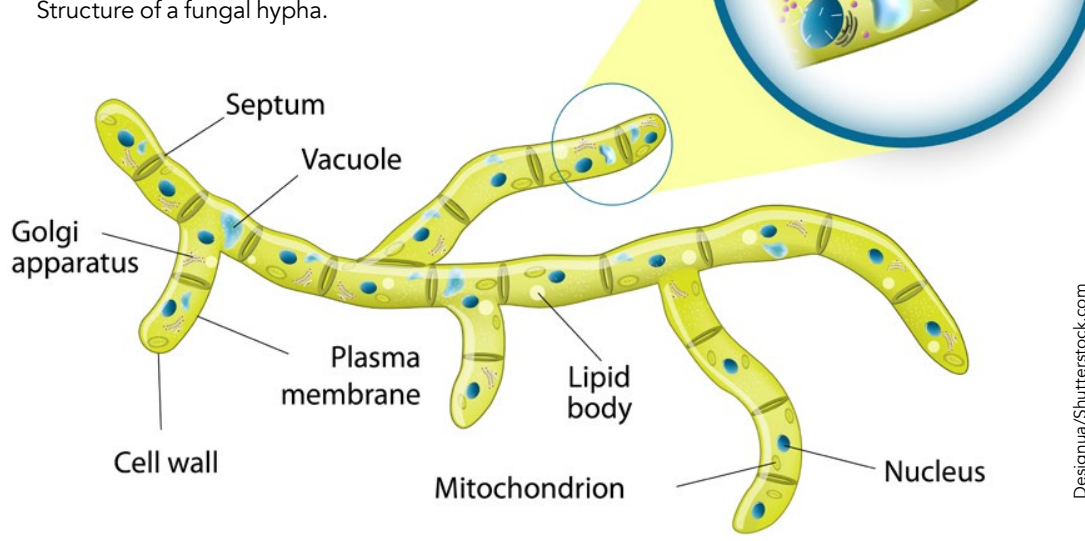
unique, much like how no two humans are exactly alike. Although the fungus does not process information the same way a human does, the individual mycelium responds to environmental stimuli in a similar fashion.

Pathogenic fungal mycelia also respond to their environment when they invade 


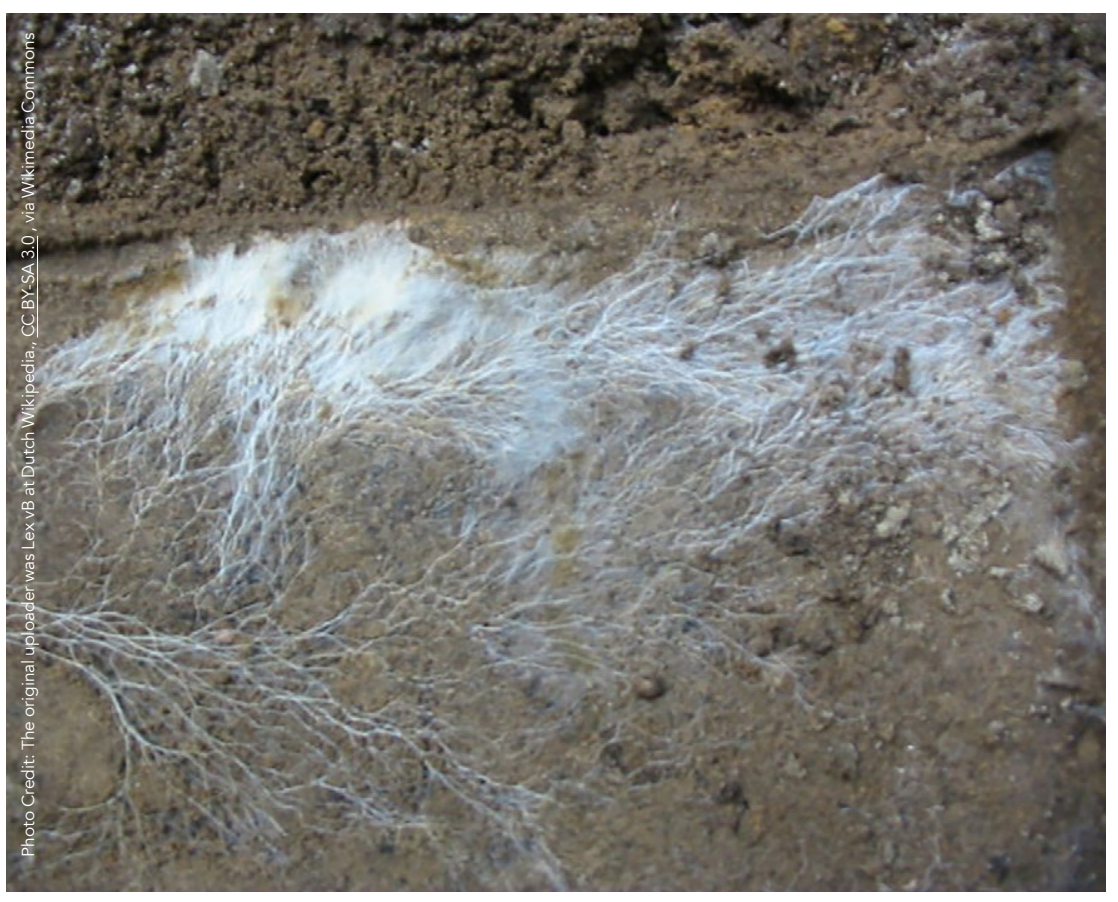

Interweaving patterns of mushroom filaments

have been shown to modify their growth form to become more invasive as the infection develops. These responses are behaviours, but the fungus is able to grasp things about its environment and show something similar to the classical conditioning of Pavlov's dogs.

In more recent experiments, some mycologists (fungal biologists) have been experimenting with fungal memory. When colonies of one species of fungus were exposed to high temperatures, they were better able to cope with a second heat shock compared to This cellular adaptation lasted up to twelve hours. Another experiment demonstrated fungal spatial awareness by saturating a wooden block with fungal mycelium and placing it on a soil tray. As the fungus grew across the soil, it reacted when it found a fresh wood block by growing all around it and invading the new food source. Scientists then moved the initial wooden block to a new soil tray tearing the fungus away from the soli. When the hyphae in the wooden block began to regrow, they extended from for original side from which they had found the second wooden block. This rudimentary memory can be

TYPES OF CONSCIOUSNESS The acute sensitivity of the fungi observed at the cellular level argues for the presence of a mind. Just like the of, and responds to, its environment. Awareness is not a unique privilege of the human mind, but it exists in many forms throughout nature. By extension, fungal consciousness, like human consciousness, is just one expression of a diverse and intricate biological phenomenon. The field of mycology has not previously applied a theory of the fungal brain to its environment.

this new framework has the potential to wider concept of consciousness. Increasingly, scientists are defining consciousness in terms of an organism's awareness and reactions to its surroundings. The organism must be able to detect things that are good for it, such as light, food, or mates, and avoid those which harm it, like toxins or predators. These are all characteristics exhibited by single cells. Even more
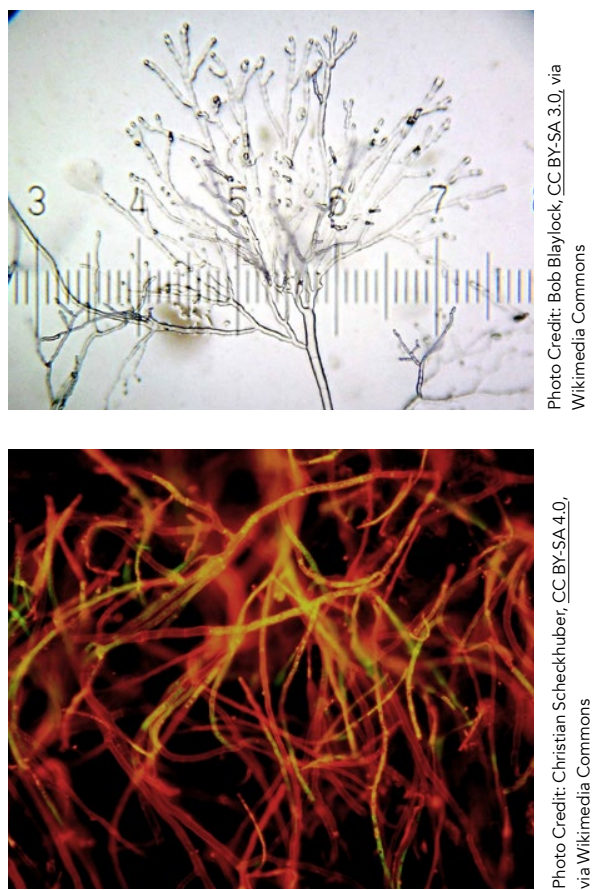

a greater level of selfhood compared to a single specialised cell within a multicellular organism. A human heart cell cannot break off and become autonomous and self-sufficient, and larger body. Therefore, a fungus or an amoeba is in theory as autonomous and self-reliant as a human.

Fungal mycelia form networks in the so which intercon mect with plants and other fungi. Professor Money claims that the of the conscingal hyphae is evidence of the consciousness of a single hypha; when these filaments ust like the animal brain, the fungal are linked in ans mind is aware of, and responds to, elevated to the lev colonies share enrich how we understand fungi and the transport through the cells, and

exchange information about their environment. Like the human brain, these networks importantly become more than the sum of their parts. This new understanding of the intricate sensory and response mechanisms serves as a vital reminder at a time of time of ecological deterioration and human hubris that we are not the only conscious organisms on the planet,

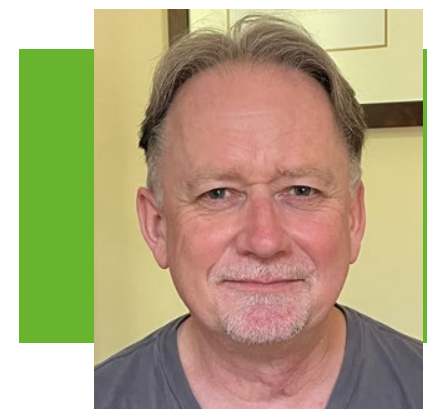

\section{Behind the Research} Nicholas P. Money

E: moneynp@miamioh.edu T: (513) 280-1160 W: www.themycologist.com its survival depends on being part of a

Research Objectives

Professor Nicholas Money is exploring microbial cognition

\section{Detail}

Address: Western Program, Miami University, Oxford, Ohio 45056, USA

Bio

Nicholas Money is a charismatic teacher, international expert on fungal biology, and the author of popular science of Biology and Western Program Director at Miamir University in Oxford, Ohio.

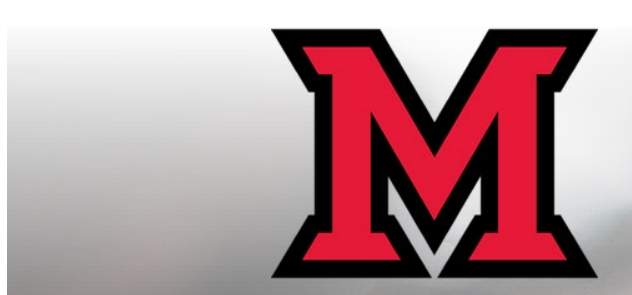
of brainless organisms is humbling. It

\section{References}

Money, N. P. (2021). Hyphal and mycelial consciousness: The concept of the fungal mind. Fungal Biology, 125, $257-259$.

Glasgow, R. D. V. (2020). Minimal selfhood. Journal of Neurogenetics, 34 (1), $198-202$

Pagan, O. R. (2019). The brain: a concept in flux. Philosophical Transactions B, 374(20180383), $1-12$.

Reber, A. R. (2016). Caterpillars, consciousness and the origins of mind. Animal Sentience, 11 (1), 1 - 13.

Gibbs, D., \& Dellinger, O.P. (1908). The daily life of Amoeba proteus. The American Journal of Psychology, 19 (2) 232-241.

\section{Personal Response}

Much of this research explores how fungi respond to Is it possible, in your opinion, for a fungus to be aware

III If we include self-awareness in the criteria of consciousness, a fungus seems to fail, but we need to push a little further and consider what we mean by selfawareness. When a fungal colony reacts to personal injury, its response is an unambiguous act of self-preservation. The fungus senses that it has been damaged and mobilises its onboard repair mechanisms, which is an expression of self-awareness. Although human self-awareness is a richer phenomenon, it must be produced by the same Consciousness is a characteristic of life, novery species. Consciousness is a characteristic of life, not a special
feature of Homo sapiens.

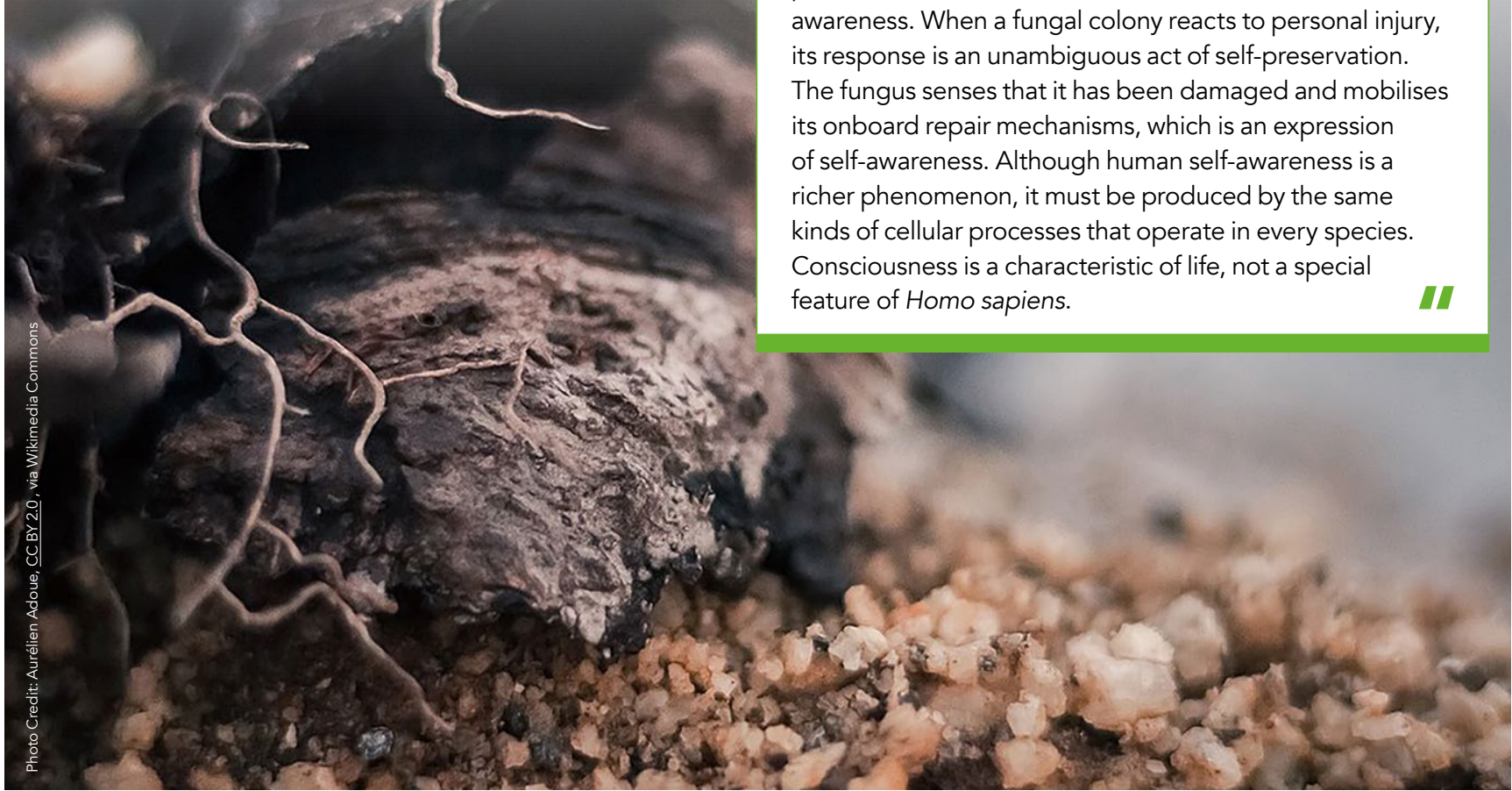
stimuli, but this is only one definition of consciousness. 